\title{
Variation of Heat Flux at Lower Frequencies of Vibration in a Vibrated Granular Bed
}

\author{
Nadeem Ahmed Sheikh, ${ }^{1}$ Shehryar Manzoor, ${ }^{2}$ and Muzaffar Ali ${ }^{2}$ \\ ${ }^{1}$ Department of Mechanical Engineering, Mohammad Ali Jinnah University, Islamabad 44000, Pakistan
}

${ }^{2}$ Department of Mechanical Engineering, UET Taxila, Taxila 47080, Pakistan

Correspondence should be addressed to Nadeem Ahmed Sheikh; ndahmed@gmail.com

Received 12 November 2014; Revised 13 February 2015; Accepted 8 March 2015

Academic Editor: Gayanath Fernando

Copyright (C) 2015 Nadeem Ahmed Sheikh et al. This is an open access article distributed under the Creative Commons Attribution License, which permits unrestricted use, distribution, and reproduction in any medium, provided the original work is properly cited.

Granular flows in vibrated bed exhibit various physical phenomena primarily driven by vibrating base. As the vibrating surface is the only source of energy in an otherwise dissipative flow, most of the theoretical models relate the steady state energy input to the RMS velocity of vibration. Here variation of heat flux is studied at varying frequency of vibration while keeping the RMS vibration velocity and the cell loading constant. Using single particle analysis and MD simulations, an extended version of grain-base collision is observed resulting in the reduction of heat flux at lower frequencies $(<50 \mathrm{~Hz})$ of vibration. The presented findings are important as most experimental studies are reported at these frequencies of excitation.

\section{Introduction}

Granular flows in the arrangement of a vibrated bed exhibit various behaviors primarily depending on the strength of vibration [1-5]. At sufficiently higher levels of acceleration the gaseous or rapid motion of granular material is observed. Such kind of flows is of greater interest from the aspect of modeling using kinetic theory models and continuum order descriptions [6,7]. The primary assumption in almost all of these models is the instantaneous nature of the dissipative collisions between grains (e.g., [8-11]). Moreover, substantial amount of work can also be found on the simulation of rapid granular flows using kinetic theory models especially for dilute and nearly elastic flow nature [12, 13]. Keeping in mind the geometry of the system, energy to sustain such flows comes from the vibrating surface. The amount of energy input is related to the nature of interaction between the grains and oscillating base.

Thus the modelling of such flows also involves a reliable and robust model for energy influx. Mostly expressions on the boundary conditions are modelled using first principals of momentum and energy balances at the surface [14]. Through various methods of averaging, conditions have been obtained that apply to two-dimensional systems of identical disks or three-dimensional systems of identical spheres that interact with smooth bumpy boundaries [15]. On average the heat flux imparted by the vibrating surface is linked with the RMS velocity of the surface [15-17] and most of the studies rely on the presumption that the frequency of vibration is uncorrelated with the mean free time of the grains $[18,19]$.

However a recent study has shown that as the base time period approaches the duration for a typical grain-base collision the amount of steady state heat flux may reduce drastically [20]. This leads to breakdown of instantaneous binary collision models. Away from this region, the effect of frequency of vibration is usually assumed to be irrelevant. Still an influence of base time period and its phase has been observed in some of the experimental observations conducted at lower frequencies of vibrations. For instance, the generation and propagation of acoustic/pressure wave have been observed on a periodic or cyclic basis in a vibrated bed $[7,19]$. Moreover, an additional mechanism of bulk heat transport has also been proposed by [19] at lower frequencies of vibration $(\mathrm{O}(10))$. Although the mechanism and effect of tapping motion are quite well understood [7, 19], still it remains to be seen how the heat flux varies in these frequencies. 
As the upper limit of the frequency spectrum has been identified, the effort of this work is to look at the lower end of the spectrum as well. This research work studies the effect of frequency of vibration on the heat flux primarily at intermediate to lower ranges of frequencies. It is important to mention that a number of experimental studies related to vibrated granular bed have been reported with frequency of oscillation of the order $\mathrm{O}(10)[16,19]$. Therefore estimation of low frequency influence on the amount of heat flux imparted in the bed is important.

\section{System Description}

The system under consideration is a vertically vibrated dry granular bed in the presence of gravity. The idea is to simulate the system that has been studied previously [20]. Therefore the predictions made here can also be verified experimentally if conducted over the range of frequencies. For the sake of completeness summarized detail of the setup is presented below.

The vibrated cell is of $18 \mathrm{~mm}$ diameter filled with 40 grains (single static layer). Each grain is of uniform radius $r=$ $2.3 \mathrm{~mm}$ with mass of $m=6.74 \mathrm{mg}$. The frequency of vibration is varied from $15 \mathrm{~Hz}$ to $20 \mathrm{kHz}$ while keeping the RMS base velocity constant at $v_{b}=0.54 \mathrm{~m} / \mathrm{s}$. At such velocity the granular material is expected to remain sufficiently fluidized.

2.1. Molecular Dynamic Simulations. The simulations are carried using discrete element modeling based on the soft sphere model using the normal force law developed by Kuwabara and Kono [21] as given by

$$
F=K \delta^{3 / 2}+\gamma \delta^{1 / 2} \frac{d \delta}{d t}
$$

where $F$ is the contact force between the colliding spheres while $\delta$ is the resultant deformation produced [21]. This requires stiffness constant $K$ and damping coefficient $\gamma$. Here $K$ is based on the experimentally measured value of $1.47 \times$ $10^{7} \mathrm{Nm}^{-3 / 2}$ reported by [20], while damping coefficient is evolved using a simulation carried out between two identical grains (for grain-grain collision) of the above-mentioned properties. The interaction between the grains is carried out at RMS base velocity while the damping coefficient is allowed to vary so as to achieve the coefficient of restitution as reported by [20]. Similar procedure was adopted for grainbase and grain-side wall collisions also. For the sake of reference the reported coefficients of restitutions are 0.68 (grain-grain), 0.74 (grain-side wall), and 0.71 (grain-base).

For each simulation grains were inserted randomly in the cell with random velocity allocations. Initial run of $10^{6}$ total collisions was performed in each case to take care of the allocation bias of initial configuration if present. The reported results are of minimum $10^{6}$ grain-base collisions with at least 250 cycles of base vibrations for each run. Time period for all simulations was kept constant at 100th of the time consumed by a typical Hertzian grain-base collision [20].

2.2. Variation of Heat Flux with Frequency. The simulations at a total of 16 different frequencies of vibration ranging from

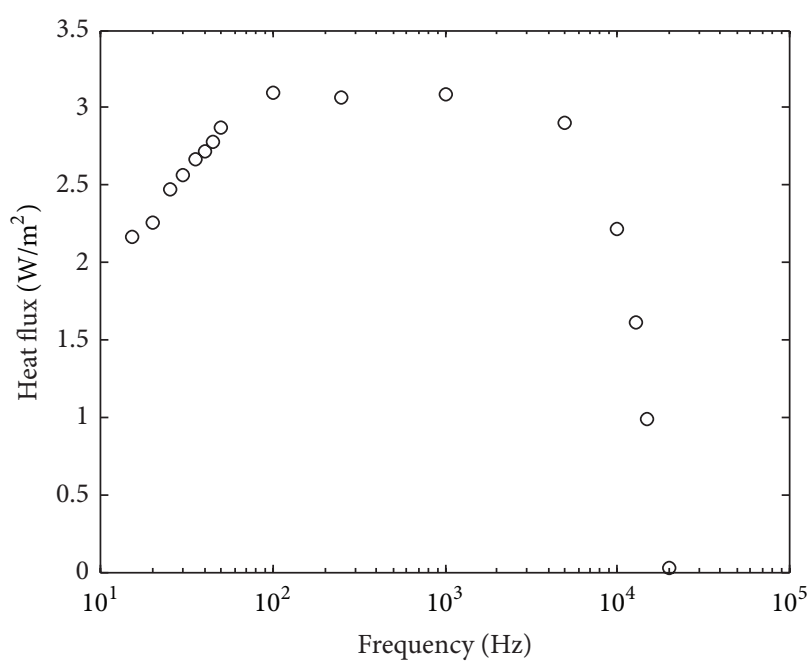

FIGURE 1: Variation of steady state heat flux at different frequencies of excitation while RMS base velocity is kept constant at $v_{b}=0.54 \mathrm{~m} / \mathrm{s}$.

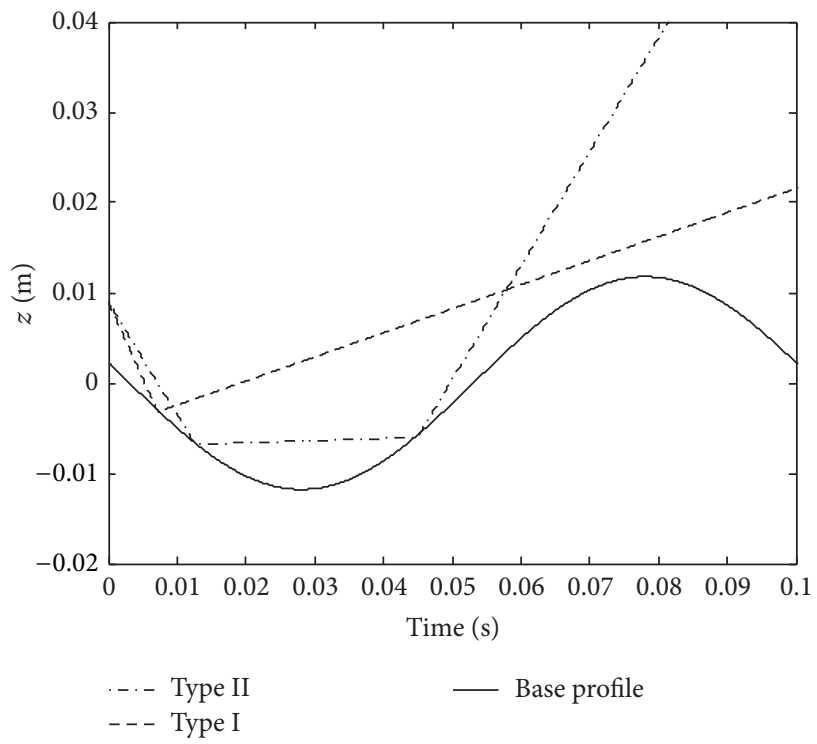

FIGURE 2: Typical types I and II grain-base collisions.

$15 \mathrm{~Hz}$ to $2.0 \mathrm{kHz}$ are carried out at fixed RMS base velocity of $v_{b}=0.54 \mathrm{~m} / \mathrm{s}$. The resultant variation of steady state heat flux injected by the base into the granular bed is shown in Figure 1. The figure shows that for the frequency varying from $50 \mathrm{~Hz}$ to $5 \mathrm{kHz}$ the base heat flux remains almost constant as expected with constant RMS velocity. With frequency excess of $5 \mathrm{kHz}$, a rapid decrease in the heat flux is observed reducing to almost zero at $20 \mathrm{kHz}$. As noted by [20], at this frequency range the time duration for a typical grain-base collision is of the same order as the time period of oscillations. Thereby the assumptions and consequences of instantaneous and binary collisions no longer remain valid.

In addition a secondary loss of heat flux is observed in the region of lower frequency oscillation $(<50 \mathrm{~Hz})$. Quite clearly the rate of decrease of heat flux is not as steep as seen at other end of frequency spectrum. However a significant percentage 

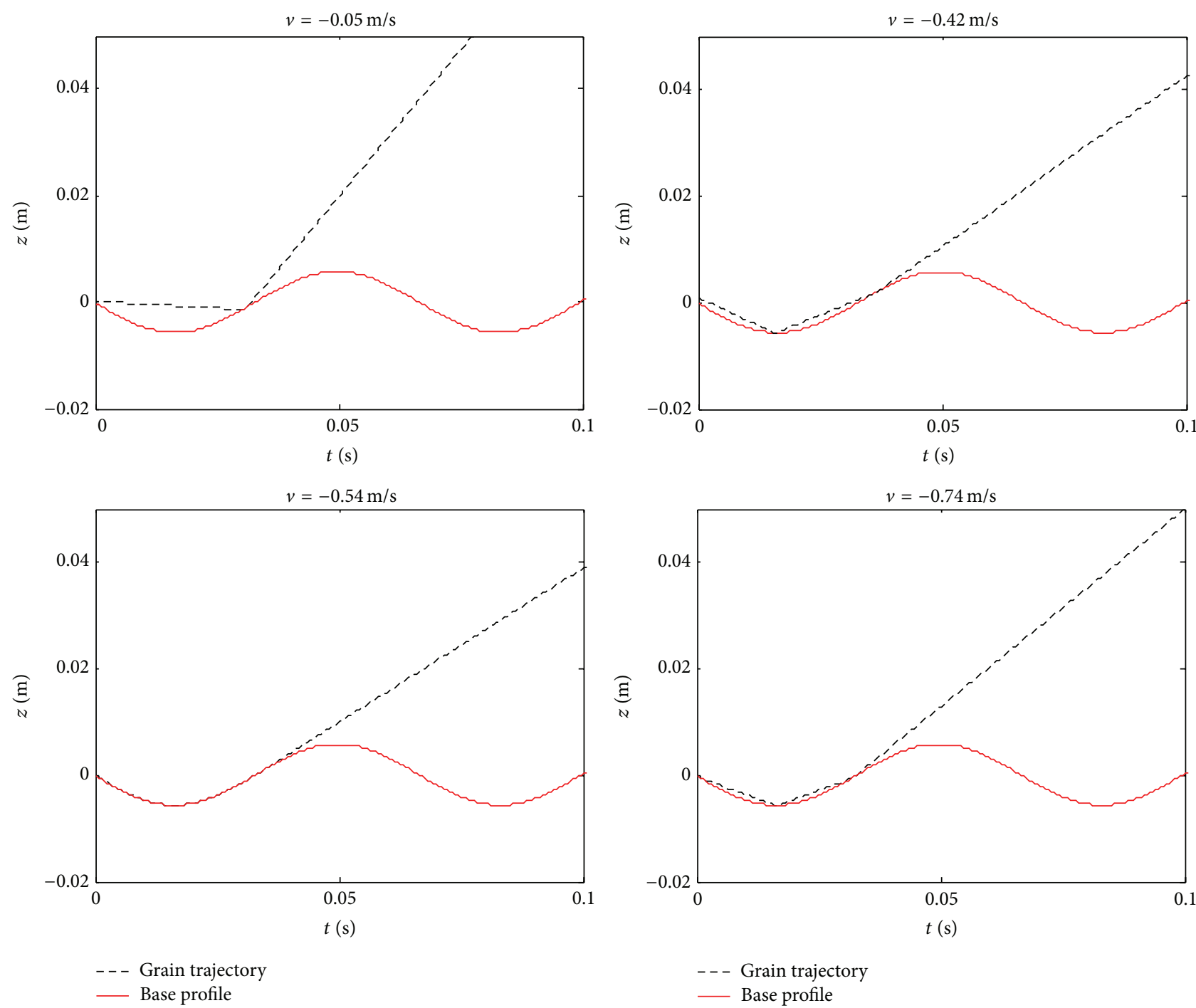

FIGURE 3: Profiles of a single grain-base interaction at varying incoming grain velocities $(v)$ at phase angle of 0.

(around 30\%) decrease of heat flux is observed. Keeping in mind that this is the region of most experimental observations (e.g., [19]) made on granular flows using vibrated beds, a detailed analysis of this incline is required. Moreover the phase of vibration is also expected to be an important parameter in this region as the time period of vibration becomes relatively large.

2.3. Single Particle Interaction with Base. In order to understand the nature of grain-base interaction in the lower frequency spectrum a more simplistic analysis is carried out following the approach used by [20]. The analysis studies the trajectory of a single particle released from a reference position with initial velocity. This allows the calculation of the grain-base interaction and an estimate of resultant coefficient of restitution from the interaction. For the finite time interaction, the equation of motion of a single particle undergoing
Hertzian collision with the moving base can be modeled using [20]

$$
\frac{d^{2} z_{c}}{d t^{2}}=\frac{K}{m}\left(z_{b}-z_{c}+r\right)^{3 / 2} H\left(z_{b}-z_{c}+r\right),
$$

where $z_{c}$ is the position of the centre of the mass of the particle and $z_{b}$ is the location of the base given by

$$
z_{b}=-a_{o}(\sin (\omega t+\phi)-1) \text {. }
$$

The same values of $m, r$, and $K$, representing mass, radius, and Hertzian stiffness coefficient of the particle, were used as detailed above. Here $t$ is the time while $\omega$ is the frequency of vibration and $\phi$ represents phase angle of the base.

In (2), $H(z)$ is a Heaviside step function given by

$$
H(z)=\left[\begin{array}{ll}
1.0, & z \geq 0 \\
0.0, & z<0
\end{array}\right] .
$$




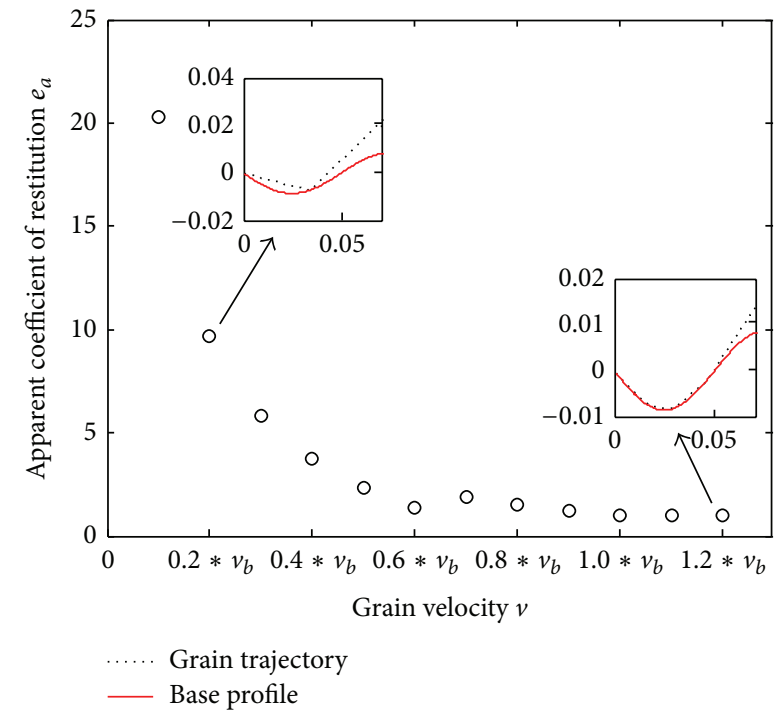

FIGURE 4: Variation of apparent coefficient of restitution at different incoming velocities in terms of base velocity $\left(v_{b}\right)$.

Note that the second order nonlinear equation (1) assumes perfectly elastic interaction in the absence of gravity. It is assumed that gravity plays no major part during the collision of grain with the base [20]. Introducing $z=z_{c}-r$, (2) can be rewritten as

$$
\frac{d^{2} z}{d t^{2}}=\frac{K}{m}\left(z_{b}-z\right)^{3 / 2} H\left(z_{b}-z\right) .
$$

Here $z$ effectively represents a zero size particle, assumed for the sake of better representation, while plotting the trajectory of grain alongside the base profile. Typically two types of collisions are observed when grain interacts with base. Figure 2 shows the typical type I and type II collisions [16] for representation at arbitrary incoming grain velocities. The type I collision involves only single interaction with base while type II has secondary interaction with the base while it is coming up. Details of both types of interactions and associated energy transfer can be found in [16].

It is important to mention that using a similar analysis [20] has recently showed that at higher frequencies of vibration the type of grain-base interaction may change quite significantly. At higher frequencies of vibration a sudden drop of flux observed in Figure 1 is linked with the breakdown of instantaneous collision approximation.

Moving to the other end of frequency spectrum, Figure 3 shows a plot of grain approaching the base at starting phase of $\phi=0.0$ and initial height of $z=0$. With increase in the incoming velocity of grain, the grain-base interaction shifts from type I to type II collision. However as the incoming velocity approaches the base velocity, type II collision converts into an "extended collision" involving multiple and sustained interactions. In addition, the effect of extended type II collision is quite remarkable on the amount of energy input into the system.
Following the approach of [20], an apparent coefficient of restitution can be defined as

$$
e_{a}=-\frac{v^{+}}{v^{-}}
$$

where $v^{+}$and $v^{-}$represent the sign of the grain velocity based on its direction or the post- and precollision velocities at $z=$ 0 . As mentioned earlier the actual coefficient of restitution for this analysis is set to unity, whereas the apparent coefficient of restitution (6) represents the measure of energy transfer into the system. Figure 4 presents the variation of $e_{a}$ with incoming velocity of grain in terms of the base velocity $v_{b}$. As evident from the figure, the apparent coefficient of restitution decreases remarkably as the incoming grain velocity approaches the base velocity. The extended type II collision is set in at $0.6 v_{b}$ and energy input is significantly reduced even at relatively higher incoming grain velocities. This suggests that higher incoming grain velocity does not mean higher energy influx as noted by [12].

While such extended type II collision can be expected at different frequencies and at different phase angles, however, at higher frequencies the chance of occurrence is quite slim due to short wavelength of vibration and the relative random position of phase of the base with respect of the incoming grain. On the other hand, at lower frequencies the wavelength increases quite significantly and the vibration phase becomes important as observed by [19].

Using MD simulations a sneak view can be obtained on the type of collisions grains experience with the base. As the region of interest is of lower frequency of vibration, a snapshot of trajectory of all grains and base is presented in Figure 5. At $15 \mathrm{Hza}$ number of multiple and repeated extended type II grain-base collisions is observed. Figure 5(a) shows the trajectories of all simulated particles. The particles are presented here as point particles for the sake of representation. Figure 5(b) is a subset of Figure 5(a) with only a handful of collisions separated here to show the presence of extended type II collisions observed using MD simulations. Quite clearly the extended type of collision involves multiple collisions and grain motion along the base as it moves upwards. For such a collision nature, the approximation of instantaneous collision is quite unlikely to hold.

The analysis carried out and the results of MD simulations suggest that the extended type II collisions are a possible cause of reduction in the heat flux injection at lower frequencies of excitation. As observed that some of these collisions are noninstantaneous in nature, therefore the relationships for the prediction of heat flux boundary conditions based on the instantaneous approximation are likely to predict different results compared to the simulated data. Therefore the estimation of heat flux while modelling granular flow at lower frequencies of vibration using principles of instantaneous binary collision may no longer give accurate results.

\section{Conclusion}

The interaction of grain-base is the source of fluidization in a vibrated granular bed. Current models provide an extensive 


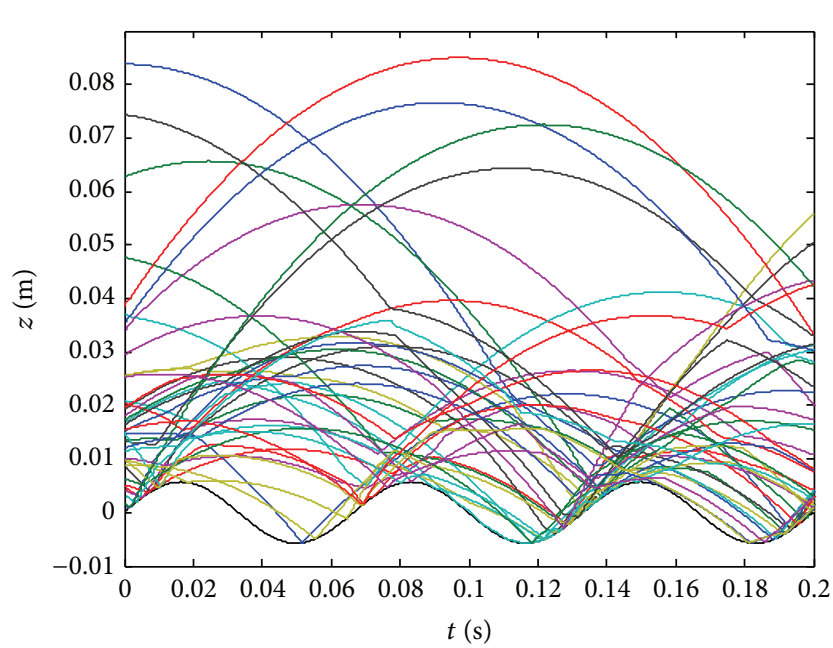

(a)

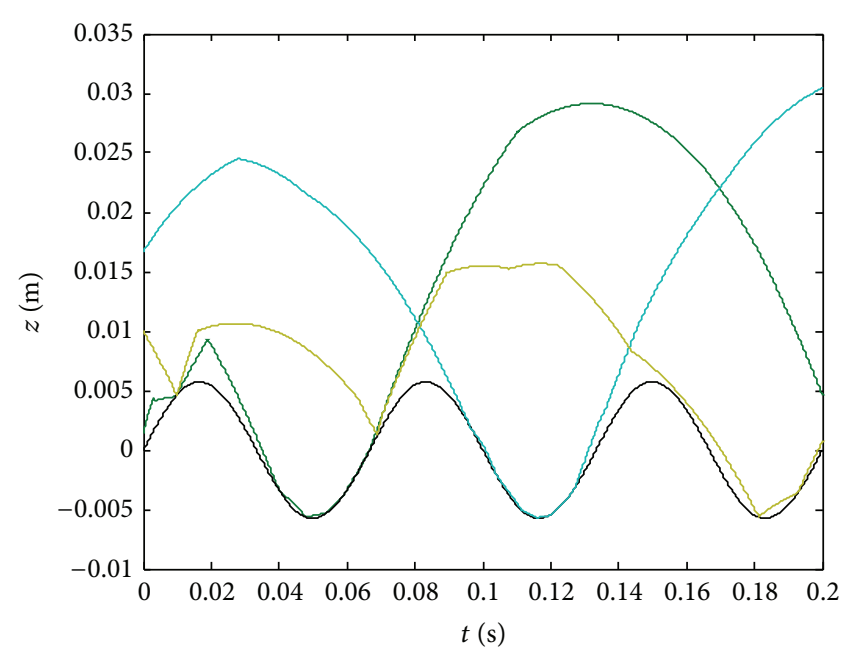

(b)

FIGURE 5: (a) A snapshot of locations of all grains and base at different instances of time during fluidization at $15 \mathrm{~Hz}$. Black solid line represents the location of base while trajectories of all particles are represented as colored lines other than black. (b) A subset of (a) with representation of trajectories of three extended type II collisions at $15 \mathrm{~Hz}$.

insight into the nature of interaction and the resultant heat flux generation. However the influence of frequency of excitation is not regarded as an influencing parameter while estimating the steady state heat flux. Using MD simulations and single particle interaction study, a detailed version of type II grain-base collisions is observed at lower frequencies of excitation. The extended type II collision involving noninstantaneous collision dynamics also limits the heat flux. With most experimental studies relying on lower frequencies of excitation, the presented results are expected to be of greater significance.

\section{Conflict of Interests}

All authors declare that there is no conflict of interests regarding the publication of this paper.

\section{References}

[1] C.-H. Liu and S. R. Nagel, "Sound in sand," Physical Review Letters, vol. 68, no. 15, pp. 2301-2304, 1992.

[2] H. K. Pak and R. P. Behringer, "Surface waves in vertically vibrated granular materials," Physical Review Letters, vol. 71, no. 12, pp. 1832-1835, 1993.

[3] E. C. Rericha, C. Bizon, M. D. Shattuck, and H. L. Swinney, "Shocks in supersonic sand," Physical Review Letters, vol. 88, no. 1, Article ID 014302, 4 pages, 2001.

[4] E. Khain and B. Meerson, "Onset of thermal convection in a horizontal layer of granular gas," Physical Review E, vol. 67, no. 2, Article ID 021306, 2003.

[5] J. Duran, Sands, Powders, and Grains: An Introduction to the Physics of Granular Materials, Springer, Berlin, Germany, 13th edition, 2000.

[6] C. M. Hrenya, J. E. Galvin, and R. D. Wildman, "Evidence of higher-order effects in thermally driven rapid granular flows," Journal of Fluid Mechanics, vol. 598, pp. 429-450, 2008.
[7] J. Bougie, S. J. Moon, J. B. Swift, and H. L. Swinney, "Shocks in vertically oscillated granular layers," Physical Review E: Statistical, Nonlinear, and Soft Matter Physics, vol. 66, no. 5, Article ID 051301, 2002.

[8] C. K. K. Lun, S. B. Savage, D. J. Jeffrey, and N. Chepurniy, "Kinetic theories for granular flow: inelastic particles in Couette flow and slightly inelastic particles in a general flowfield," Journal of Fluid Mechanics, vol. 140, pp. 223-256, 1984.

[9] J. T. Jenkins and M. W. Richman, "Grad's 13-moment system for a dense gas of inelastic spheres," Archive for Rational Mechanics and Analysis, vol. 87, no. 4, pp. 355-377, 1985.

[10] J. Ding and D. Gidaspow, "A bubbling fluidization model using kinetic theory of granular flow," AIChE Journal, vol. 36, no. 4, pp. 523-538, 1990.

[11] V. Garzó, J. W. Dufty, and C. M. Hrenya, "Enskog theory for polydisperse granular mixtures. I. Navier-Stokes order transport," Physical Review E. Statistical, Nonlinear, and Soft Matter Physics, vol. 76, no. 3, Article ID 031303, 27 pages, 2007.

[12] T. W. Martin, J. M. Huntley, and R. D. Wildman, "Hydrodynamic model for a vibrofluidized granular bed," Journal of Fluid Mechanics, vol. 535, pp. 325-345, 2005.

[13] H. Viswanathan, N. A. Sheikh, R. D. Wildman, and J. M. Huntley, "Convection in three-dimensional vibrofluidized granular beds," Journal of Fluid Mechanics, vol. 682, pp. 185-212, 2011.

[14] J. T. Jenkins and M. W. Richman, "Boundary conditions for plane flows of smooth, nearly elastic, circular disks," Journal of Fluid Mechanics, vol. 171, pp. 53-69, 1986.

[15] J. T. Jenkins and M. Y. Louge, "On the flux of fluctuation energy in a collisional grain flow at a flat, frictional wall," Physics of Fluids, vol. 9, no. 10, pp. 2835-2840, 1997.

[16] S. Warr and J. M. Huntley, "Energy input and scaling laws for a single particle vibrating in one dimension," Physical Review E, vol. 52, no. 5, pp. 5596-5601, 1995.

[17] M. W. Richman, "Boundary conditions for granular flows at randomly fluctuating bumpy boundaries," Mechanics of Materials, vol. 16, no. 1-2, pp. 211-218, 1993. 
[18] V. Kumaran, "Dense granular flow down an inclined plane: from kinetic theory to granular dynamics," Journal of Fluid Mechanics, vol. 599, pp. 121-168, 2008.

[19] J. M. Huntley, T. W. Martin, M. D. Mantle et al., "NMR measurements and hydrodynamic simulations of phase-resolved velocity distributions within a three-dimensional vibrofluidized granular bed," Proceedings of the Royal Society A: Mathematical, Physical and Engineering Sciences, vol. 463, no. 2086, pp. 25192542, 2007.

[20] J. M. Huntley, T. Tarvaz, M. D. Mantle et al., "Nuclear magnetic resonance measurements of velocity distributions in an ultrasonically vibrated granular bed," Philosophical Transactions of the Royal Society A, Article ID 20130185, 2014.

[21] G. Kuwabara and K. Kono, "Restitution coefficient in a collision between two spheres," Japanese Journal of Applied Physics, Part 1: Regular Papers \& Short Notes, vol. 26, no. 8, pp. 1230-1233, 1987. 

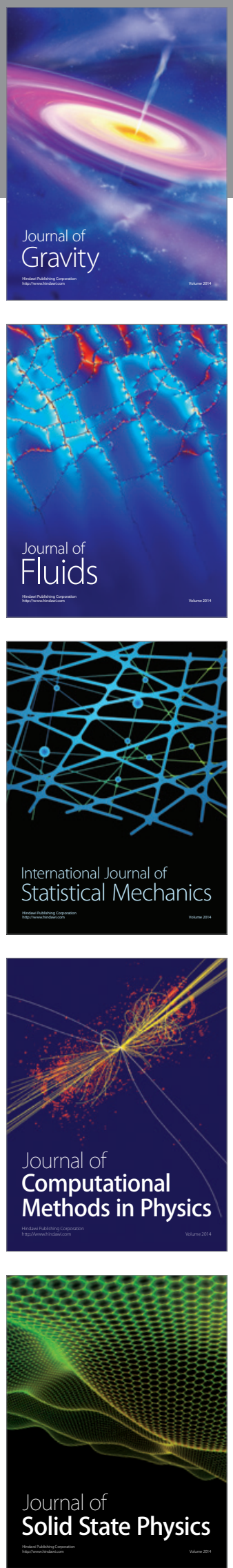

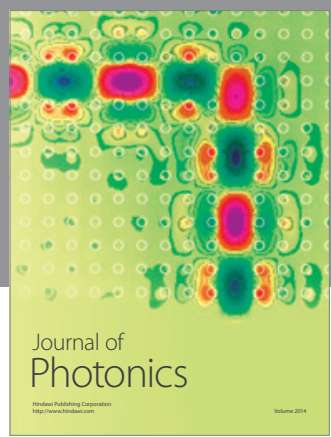

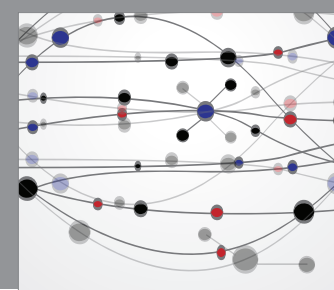

The Scientific World Journal

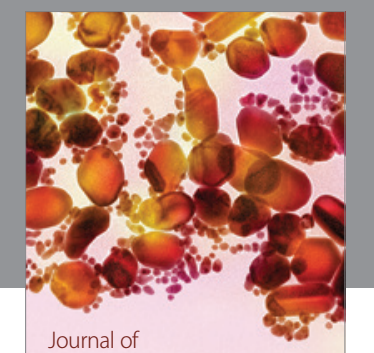

Soft Matter
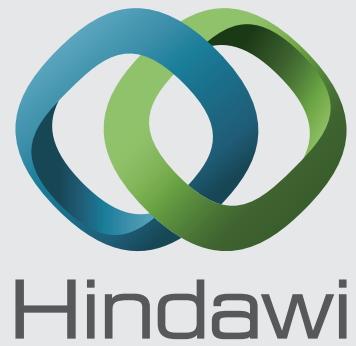

Submit your manuscripts at

http://www.hindawi.com
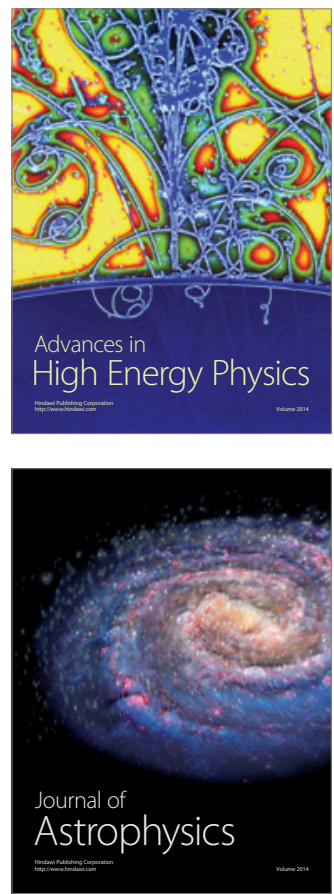
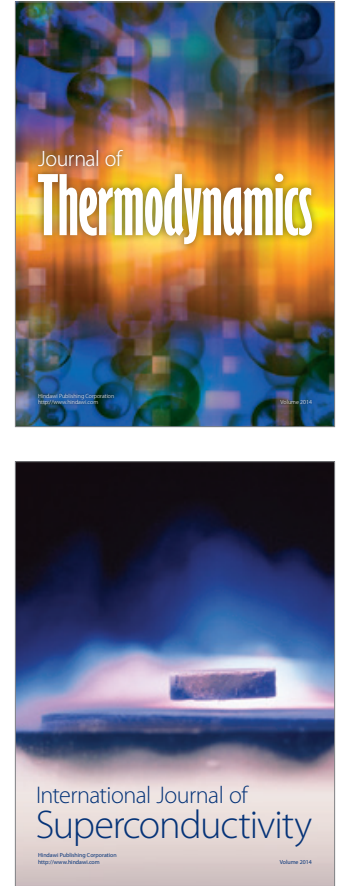
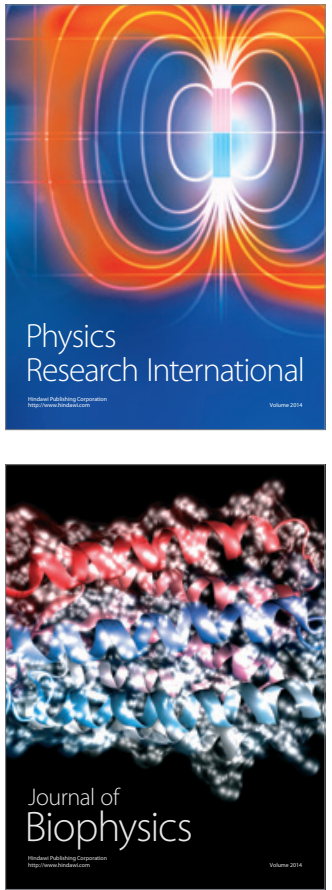
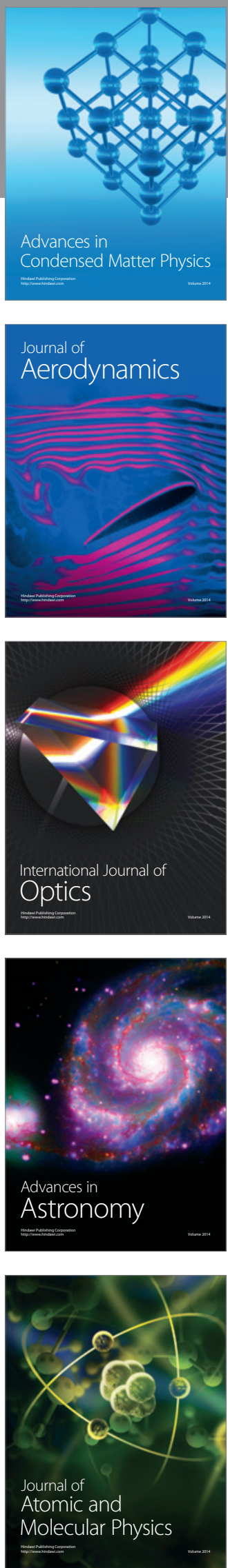